\title{
(18F)-FDG PET IMAGING AND NEURO- OPHTHALMOLOGICAL FINDINGS IN DIAGNOSIS OF PROGRESSIVE SUPRANUCLEAR PALSY: CASE REPORT
}

\author{
Ara Kaprelyan', Alexandra Tzoukeva', Pavel Bochev², Borislav Chaushev ${ }^{2}$, \\ Margarita Grudkova ${ }^{1}$ \\ ${ }^{1}$ Department of neurology and neuroscience, \\ ${ }^{2}$ Department of nuclear medicine and radiotherapy \\ Medical University "Prof. Dr. P. Stoyanov", Varna
}

\begin{abstract}
INTRODUCTION: Progressive supranuclear palsy is a neurodegenerative disorder, described as a tauopathy. The neuroimaging and visual symptoms are among the most important in the diagnosis of this disease. The ophthalmoparesis mainly concerns voluntary eye movements. Patients mostly present with vertical palsy, involuntary eye movements, convergence insufficiency, and diplopia.

CASE REPORT: A 62-year-old female patient was admitted to the hospital because of the gait disturbance and frequent falls. She had impaired memory, handwriting and speech for one year. On neurological and neuro-ophthalmological examinations, the patient presented with vertical gaze palsy, involuntary eye movements, dysarthria, tremor in the right hand, and cerebellar ataxia. Blood tests were normal. (18F)-FDG PET revealed bilateral zones of moderate hipometabolism in the regions of posterior frontal cortex, anterior cingulum, left thalamus, head of left striatum, and left temporal lobe. Neuropsychological tests revealed cognitive impairment (MMSE-26; IADL-1).

CONCLUSION: Based on the literature review and our own notices, we suggest that (18F)-FDG PET scanning and neuro-ophthalmological examination in addition to structural neuroimaging and clinical findings play a crucial role in the differential diagnosis of PSP and other parkinsonian syndromes.
\end{abstract}

Keywords: PSP, (18F)-FDG PET, visual symptoms, differential diagnosis

Address for correspondence:

Prof. Ara Kaprelyan, $M D, P h D$,

Head of Department of neurology and neuroscience,

"Prof. Dr. P. Stoyanov" Medical University of Varna,

55 M. Drinov str.

9002 Varna, Bulgaria

phone: + 359888745562

e-mail:arakapri07@yahoo.co.uk

Received: November 12, 2014

Accepted: December 19, 2014

\section{INTRODUCTION}

Progressive supranuclear palsy (PSP), known as Steele-Richardson-Olszewski syndrome is a neurodegenerative disease, described as a tauopathy $(4,14)$. The cardinal clinical characteristics include supranuclear dysfunction accompanied by extrapyramidal symptoms (Parkinsonism), cognitive impairment, gait disturbance, and frequent falls $(2,5,10)$. The principal cerebral areas of the brain affected are the basal ganglia (subthalamic nucleus, substantia nigra, and globus pallidus), brainstem (midbrain), 
frontal cerebral cortex, and the cerebellum (dentate nucleus).

According to the literature, the differential diagnosis with parkinsonian syndromes is based on the neurological, neuro-ophthalmological, and neuroimaging findings $(1,3,6,8)$. It is well-known that the visual symptoms are among the most important in the diagnosis of PSP. The ophthalmoparesis mainly concerns voluntary eye movements. Clinical data support the notion that patients may present with vertical palsy, involuntary eye movements called "square-wave jerks", convergence insufficiency, and diplopia (double vision) $(2,5,15)$. In addition, structural and functional neuroimaging is an important marker for the differential diagnosis of PSP, idiopathic Parkinson's disease, and other parkinsonian syndromes $(7,9,11,13)$. Respectively, a large group of (18-FDG)-PET studies reveal decreased glucose metabolic activity in bilateral frontal cortex, striatum, and cerebellum $(1,3,6,12,15)$.

In accordance with previously reported observations, we describe a case of PSP and try to assess the discriminative power of neuro-ophthalmological and (18-FDG)-PET findings to differentiate this serious movement disorder from other parkinsonian syndromes.

\section{CASE REPORT}

A 62-year-old female patient was admitted to the hospital because of the gait disturbance that caused imbalance and frequent falls with traumatic injuries. She had impaired memory, handwriting and speech since one year. On neurological and neuro-ophthalmological examination, the patient presented with vertical gaze palsy, involuntary eye movements („square-wave jerks"), dysarthria, tremor in the right hand, and cerebellar ataxia. Standard blood tests were normal. MRI showed mild moderate brain atrophy. (18F)-FDG PET (Fig.1-2) revealed bilateral zones of moderate hipometabolism in the regions of posterior frontal cortex, anterior cingulum, left thalamus, head of left striatum, and left temporal lobe (old infarction in the left middle cerebral artery). Neuropsychological tests revealed mild cognitive impairment (MMSE-26; IADL-1).

\section{DISCUSSION}

Here, we present the clinical description, typical neuro-ophthalmological findings, and functional neuroimaging of a patient with progressive supranuclear palsy, identified by clinical diagnostic criteria of National Institute of Neurological Disorders and Stroke and the Society for PSP (NINDS-SPSP) (4). According to the previous reports, the early detection and differential diagnosis of this neurodegenerative disorder is based mainly on specific visual symptoms accompanied with cognitive decline and parkinsonian clinical manifestations $(2,5,9,13)$. In our case, the neuro-ophthalmological examination found vertical (up- and downward) gaze palsy and horizontal saccades (involuntary eye movements mimicking nistagmus). Recent studies reveal that certain visual symptoms accompanied with frequent falls are clinical hallmarks of the major PSP subgroup, defined as Richardson's syndrome (RS) $(1,6,8)$. Respectively, our neuro-ophthalmological findings are in contrast to the subgroup of patients who refer to PSP-parkinsonism (PSP-P), presenting primarily with asymmetric extrapyramidal symptoms (bradykinesia, rigidity, and tremor) and modest responsiveness to levodopa therapy.

Although the anatomical neuroimaging is useful in PSP diagnosis, it is generally limited to the exclusion of cerebrovascular infarction, brain tumor, lobar atrophy or other structural pathology that causes similar clinical manifestations $(4,10)$. Usually magnetic resonance imaging (MRI) shows atrophy of upper brain stem, superior cerebellar peduncles, and dilation of third ventricle. In accordance, we found moderate brain atrophy on our MRI scans.

Several works confirm the clinical usefulness of nuclear medicine techniques in PSP diagnosis and discrimination from other parkinsonian syndromes $(7,11,14)$. Respectively, (18-F)-FDG PET allows the characterization of cerebral hipometabolism in PSP patients $(3,6,9,12,15)$. In relation, our radiological findings detected similar decrease in glucose metabolic activity in bilateral posterior frontal cortex, anterior cingulum, left thalamus, head of left striatum, and left temporal lobe. These notices corresponded to major clinical features (frequent falls, mild cognitive impairment, and tremor) and neuro-ophthal- 
Ara Kaprelyan, Alexandra Tzoukeva, Pavel Bochev et al.

mological abnormalities (vertical gaze palsy and horizontal saccades) described in our case report.

Despite the recent advances in understanding of PSP pathophysiology, imaging techniques, and genetic analyses, the diagnostic accuracy of this neurodegenerative disorder remains difficult. Accordingly, we suggest that the combining of clinical, neuro-opthalmological, and radiological markers shows great potential for improvement of PSP diagnosis and its determination from other movement disorders.

\section{CONCLUSION}

Based on the literature review and our own notices, we suggest that (18F)-FDG PET scanning and neuro-ophthalmological examination in addition to structural neuroimaging and clinical findings may play a crucial role in the early recognition of patients with PSP and differential diagnosis with various parkinsonian syndromes.
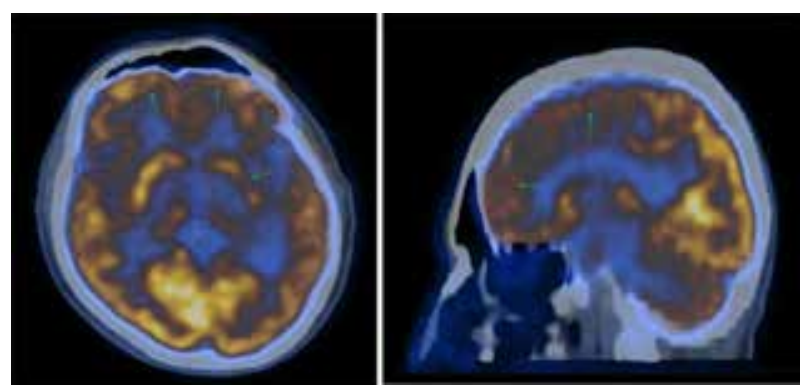

Fig. 1-2. (18F)-FDG PET reveals bilateral zones of moderate hipometabolism in the regions of posterior frontal cortex, anterior cingulum, head of left striatum, and left temporal lobe

\section{REFERENCES}

1. Akdemir Ü, A Tokçaer, A Karakuş, L Kapucu. Brain 18F-FDG PET imaging in the differential diagnosis of Parkinsonism. Clinical Nuclear Medicine, 2014;39(3):225-314.

2. Amtage F, C Maurer, S Hellwig, et al. Functional correlates of vertical gaze palsy and other ocular motor deficits in PSP: An FDG-PET study. Parkinsonism \& Related Disorders, 2014;20(8):898-906.

3. Berti V, A Pupi, L Mosconi. PET/CT in diagnosis of movement disorders. Annals of the New York Academy of Sciences, 2011;1228:93-108.

4. Borroni B, A Benussi, A Pilotto, et al. Diagnosing progressive supranuclear palsy: role of biological and neuroimaging markers. J Alzheimers Dis Parkinsonism, 2014;4:6.

5. Botha $\mathrm{H}, \mathrm{J}$ Whitwell, A Madhaven, $\mathrm{M}$ Senjem, $\mathrm{V}$ Lowe, $\mathrm{K}$ Josephs. The pimple sign of progressive supranuclear palsy syndrome. Parkinsonism \& Related Disorders, 2014;20(2):180-185.

6. Gerhard A. Imaging of neuroinflammation in Parkinsonian syndromes with positron emission tomography. Curr Neurol Neurosci Rep, 2013;13:405.

7. Hellwig S, M Reinhard, F Amtage, et al. Transcranial sonography and [18F]fluorodeoxyglucose positron emission tomography for the differential diagnosis of parkinsonism: a head-to-head comparison. Eur J Neurol, 2014;21(6):860-866.

8. Jin $\mathrm{S}, \mathrm{M}$ Oh, $\mathrm{S}$ Oh, et al. Differential diagnosis of Parkinsonism using dual-phase F-18 FP-CIT PET imaging. Nucl Med Mol Imag, 2013;47(1):44-51.

9. Srulijes K, M Reimold, R Liscic, et al. Fluorodeoxyglucose positron emission tomography in Richardson's syndrome and progressive supranuclear palsy-parkinsonism. Movement Disorders, 2012;27(1):151-155.

10. Takahashi R, K Ishii, T Kakigi, T Yokoyama, E Mori, T Murakami. Brain alterations and MiniMental State Examination in patients with progressive supranuclear palsy: Voxel-Based investigations using 18F-Fluorodeoxyglucose positron emission tomography and magnetic resonance imaging. Dement Geriatr Cogn Dis Extra, 2011;1(1):381-392.

11. Teune L, A Bartels, B Jong, et al. Typical cerebral metabolic patterns in neurodegenerative brain diseases. Movement Disorders, 2010;25(14):2395-2404.

12. Teune L, R Renken, D Mudali, et al. Validation of parkinsonian disease-related metabolic brain patterns. Movement Disorders, 2013;28(4):547-551.

13. Tripathi $M, V$ Dhawan, $S$ Peng, et al. Differential diagnosis of parkinsonian syndromes using F-18 fluorodeoxyglucose positron emission tomography. Neuroradiology, 2013;55(4):483-492.

14. Zalewski N, H Botha, J Whitwell, V Lowe, D Dickson, K Josephs. FDG-PET in pathologically confirmed spontaneous $4 \mathrm{R}$-tauopathy variants. J Neurol, 2014;261(4):710-716.

15. Zhao P, B Zhang, S Gao. 18[F]-FDG PET study on the idiopathic Parkinson's disease from several parkinsonian-plus syndromes. Parkinsonism \& Related Disorders, 2012;18(Suppl. 1):S60-S62. 